\title{
Operational Radiation Protection in High Energy Physics Accelerators. Implementation of ALARA in Design and Operation of Accelerators
}

\author{
Alberto Fassò and S. Rokni \\ SLAC National Accelerator Laboratory, 2575 Sand Hill Road, Menlo Park, CA 94025, \\ USA
}

Corresponding author: Alberto Fassò, fasso@slac.stanford.edu, phone 650-926-4762, fax: 650-925-3569

Submitted for International School of Radiation Damage and Protection, "Operational Radiation Protection for Accelerators in Research and Medicine", held in Erice, Italy, 14-20 May 2009 


\title{
Operational Radiation Protection in High Energy Physics Accelerators.
} Implementation of ALARA in Design and Operation of Accelerators

\author{
Alberto Fassò and S. Rokni \\ SLAC National Accelerator Laboratory, 2575 Sand Hill Road, Menlo Park, CA 94025, \\ USA
}

\section{Introduction}

It used to happen often, to us accelerator radiation protection staff, to be asked by a new radiation worker: "How much dose am I still allowed?" And we smiled looking at the shocked reaction to our answer: "You are not allowed any dose". Nowadays, also thanks to improved training programs, this kind of question has become less frequent, but it is still not always easy to convince workers that staying below the exposure limits is not sufficient. After all, radiation is still the only harmful agent for which this is true: for all other risks in everyday life, from road speed limits to concentration of hazardous chemicals in air and water, compliance to regulations is ensured by keeping below a certain value. It appears that a tendency is starting to develop to extend the radiation approach to other pollutants $\left({ }^{1}\right)$, but it will take some time before the new attitude makes it way into national legislations.

\section{What is ALARA?}

The acronym ALARA (As Low As Reasonable Achievable), sometimes spelled as "as Readily Achievable" $\left({ }^{2,3}\right)$, together with its predecessor ALAP (As Low As Practical $\left({ }^{4}\right)$, or Practicable $\left({ }^{5}\right)$, or Possible — note that slight semantic differences could be found between all these variants) did not have originally a very precise meaning besides the fact that no threshold had been found for harmful effects of radiation. A history of the concept is given in $\left(^{1}\right)$ and in $\left({ }^{6}\right)$.

A precise definition of the concept was eventually provided in $1977\left(^{7}\right)$ by the International Commission on Radiological Protection (ICRP). Since then, the acronym has been used in the publications of many national and international organizations such a NCRP $\left({ }^{8}\right)$ and EURATOM $\left({ }^{9}\right)$, but not in those of 
the ICRP, who systematically use the whole expression "as low as reasonable achievable, taking economic and social factors into account" $\left({ }^{10-12}\right)$.

In its Publication 26, the ICRP set forth its radiation protection philosophy based on three principles:

1) Justification: no practice shall be carried out unless its introduction produces a positive net benefit

2) Optimization: all exposures shall be kept as low as reasonably achievable, economic and social factors being taken into account

3) Limitation: the dose equivalent to individuals shall not exceed the limits recommended by ICRP

This apparently simple philosophy, now adopted worldwide by national laws and regulations, has several non obvious implications when examined in detail, and ICRP itself has being revising its overall meaning over the last 30 years. First of all, at the time ICRP was written, it was not immediately evident who is the person, or the institution, responsible for implementing the three principles. It appears now that principles 1) and 3) are essentially aimed at regulatory bodies, mainly at the national level, so we will not concern us with them here. Here we will deal with principle 2) (optimization), which is being commonly identified with the ALARA acronym, and we will see how it can be applied to the field of accelerators. This principle can involve various people at different levels. These can be legislators, but also managers, engineers and workers. The kind of optimization they can perform is different at the different levels, as we will show below.

Optimization of radiation exposures, as originally intended by ICRP, can be carried out because of the assumed lack of threshold and linearity of "detriment" (probability of radiation harmful effects) with dose equivalent. The new definition of the latter radiation quantity in $\left(^{7}\right)$ was such that complete linearity, or additivity, could be ensured also with respect to partial irradiations and mixed fields. This had not been the case previously, when the adopted protection quantity (MADE) and operational quantity (Dose Equivalent Index) were defined in a non-additive way. The protection quantity has been re-defined and re-named in subsequent ICRP recommendations, with the names of Effective Dose Equivalent and Effective Dose, but the basic full linearity of the definition has been retained and is still at the root of optimization. 
On the basis of the resulting model, known as the LNT (Linear-No Threshold) model, collective dose equivalent is a good measure [ICRP 26, para. 22] of radiation detriment (shortly, "collective dose" from now on). Optimization consists in minimizing the total cost of a design feature of a facility or of an operational procedure, where the cost is the sum of the basic production cost of the practice (excluding the cost of radiation protection), plus the cost of achieving a selected level of radiation protection, plus the cost of corresponding detriment.

Several considerations must be made about this cost-benefit analysis:

a) The result of the optimization must respect the third principle of protection: the dose limits may not be exceeded

b) A purely quantitative optimization requires that the three costs (the production and the protection cost and the cost of detriment) be measured with the same unit. For this purpose, it is necessary to assign a monetary value to the unit of collective dose (dollar per person-Sv or dollar per personrem). This value is known as $\alpha$.

c) There are aspects of the optimization which cannot be handled in a quantitative way, in particular the "economic and social factors" mentioned by ICRP.

We will examine more in detail the above three points.

Point a): in its most recent recommendations $\left({ }^{12,13}\right)$ ICRP has introduced another limiting concept: that of dose constraints. A dose constraint is an individual dose level lower that the legal limits, related to a particular radiation source, which can be set by an authority (national, or local management) to avoid that the sum of contributions from different sources exceed the limit, or based on some extra consideration such as the benefit of allowing access to non radiation workers or to the public.

Point b): who can assign a monetary value to $\alpha$ ? Certainly not the persons who will carry out the optimization. In general, it is assumed that the value be assigned by a superior authority: but the choice is very delicate, since it is implicitly tantamount to assigning a monetary value to a human life. In $\left({ }^{10}\right)$, values ranging between $\$ 1000$ and $\$ 100000$ per person-Sv are mentioned. Nowadays, higher values such as $\$ 200000$ or even $\$ 1100000$ are commonly used in US laboratories. It must be added that the importance 
of collective dose for an assessment of detriment has been weakened by an ever increasing attention to individual dose distributions, with a higher weight being attributed to individual doses close to the limits (or to the dose constraints), and a lower weight assigned to doses close to background. According to some authors $\left({ }^{14}\right)$ this does not necessarily implicitly reflect a lower trust in the LNT model as much as an increasing importance given to the individual with respect to the collectivity in modern society.

Point c): the non-numerical factors are being given an ever increasing importance by ICRP $\left({ }^{13}\right)$, so that the optimization process is gradually shifting from a pure mathematical procedure to a more complex process (often iterative) where technical, managerial and often public relation elements are combined. In particular, as we will show in the following, past experience and accumulated knowledge from similar situations are likely to carry a large weight in the final choice.

There is an aspect of the ALARA philosophy that we cannot discuss in detail here because its possible consequences depend on the different legal system of each country. To base a law on an optimization makes it difficult for a court to decide when the law has been infringed. In $\left({ }^{15}\right)$ it is said about the situation in the US: "Although a majority of courts have held that the standard of care is the federal dose limits, a small minority of courts have held that the ... ALARA regulation provides the standard of care".

In summary, three radiological protection factors can be distinguished:

1) the factors which must always be included in the analysis: the cost of protection and the collective doses

2) the factors which may not always be necessary: individual dose distribution, time distribution of doses, the population receiving the doses, the probability of events, the reliability of options, discomfort from protective clothing, etc.

3) possible non-radiological factors: e.g., aesthetic or public relation reasons, public acceptance, efficiency and planning requirements, additional non-radiological risks, etc. If there are such factors, the quantitative cost-benefit solution will not be the optimum solution. The qualitative factors must be combined with the quantitative solution to give the true optimum. This decision 
making is complex, and different persons will come to different conclusions. But it is important that the optimization process be fully documented.

\section{How ALARA can be misinterpreted}

Before getting into more detail, it is important to get rid of two opposite common misconceptions about ALARA.

Before optimization was formally introduced in 1977 in ICRP Publication $26\left(^{7}\right)$, ALARA, ALAP and similar expressions could be interpreted as "asking employers to do anything short of bankruptcy to reduce exposure" (as humorously put by R. Wilson $\left({ }^{1}\right)$ ). Without going to such extremes, it still happens to see ALARA presented as a way to reduce doses without any mention of balancing with costs. But resources spent to reduce doses need to be balanced against the detriment avoided. Reducing doses below this point results in a misallocation of resources which could be spent elsewhere and have a greater impact on health. As ICRP states in its Publication $103\left({ }^{13}\right)$, “Optimisation of protection is not minimisation of dose. Optimised protection is the result of an evaluation, which carefully balances the detriment from the exposure and the resources available for the protection of the individuals. Thus the best option is not necessarily the one with the lowest dose".

The second misconception is rooted in Publication 26 itself. In its first attempt to set up a rational basis for cost-benefit analysis, ICRP centered all optimization on collective dose. The resulting mathematical framework was elegant and apparently free from subjective biases, but failed to account for many components of the detriment which could not be expressed in a quantitative way. Since then, the Commission has gradually revised its original scheme, giving an increasing importance to the "economic and social factors", in particular the involvement of all "stakeholders" (authorities, management, staff, public) in the ALARA process. A larger weight given to individual doses has also reduced the importance of collective dose in optimization. The ALARA acronym, from which the fundamental part "economic and social factors being taken into account" has disappeared, is sometimes responsible for some misunderstanding of the full meaning of radiation protection standards.

\section{Applying ALARA to accelerator radiation protection}


As we have already mentioned, ALARA is a responsibility for everybody concerned with radiation protection, from lawmakers down to managers to machine designers to staff and contractors. For each of these categories, it implies a different kind of action. At the highest level, the result of cost-benefit analysis is likely to be embodied in the setting of dose limits and authorized limits (for instance, of radioactivity releases). For a regulatory authority, the focus is not on specific outcomes for a particular situation, but rather on processes, procedures, and judgements $\left({ }^{13}\right)$. People responsible for a particular project, such as a new accelerator site, may establish a dose constraint for each machine planned to be built on that site. The cost-benefit analysis will need to be done within these constraints as well as within budget constraints, and is likely to take into account the cost of environmental impact and other kinds of harmful effects, in addition to the radiation detriment to workers and the public. It is at this level of decision that the involvement of stakeholders is most important, for instance by means of public hearings. At the other end of the range, that of radiation workers (both staff and contractors) ALARA can be practiced in a simple way. The cost-benefit analysis is obvious when the cost is zero: so, if it is possible, a given work should be carried out far from a radioactive hot spot, and it is the responsibility of the radiation protection staff (RP) to teach this habit. Moving out of high radiation areas, increasing distance from the source and reducing time close to it have always been the very basis of radiation protection even at a time when ALARA had not yet been invented. Careful interaction between RP and workers is necessary to plan in advance work in radioactive areas: in some extreme cases a "cold rehearsal" will help to minimize doses. This kind of ALARA practice apparently does not require the kind of formal cost-benefit study which is typical of large projects, but in reality the organization of a good professional RP structure, and the training of technicians and radiation workers, have a cost which can be calculated. What is not easy, is to compare $a$ priori this cost with the doses which will be received when running the facility. Generally, this is done implicitly by comparing with the experience from similar facilities running under the same dose constraints.

At a more technical level, on which this lecture is focused, it is useful to distinguish between design and operation, although in general design choices will impose constraints on operating procedures $\left({ }^{11}\right)$. Generally, quantitative optimization is more important in the design phase, while in operation qualitative factors are more important. What is essential, for ALARA practiced at any level, is that the choices be fully 
documented together with the criteria which have brought to those choices. When the criteria are qualitative, it is more likely that subjective judgments play a large role, but those judgments must be equally recorded.

\section{Shielding design}

It is essential that shielding design and machine design be developed at the same time and that RP staff work in close collaboration with the management and with the accelerator engineers. This work involves several iterations. At the best accelerator sites, this kind of strict collaboration has always been practiced, and it is interesting to see how it agrees with the latest ICRP recommendations that "The organizational approach should include involvement and participation of all workers" $\left({ }^{13}\right)$.

One of the most critical parameters in accelerator shielding design is a good estimation of beam losses. Controlled beam losses, such as those expected to occur on collimators and beam dumps, can be estimated rather accurately, but it is much more difficult to predict random losses. Several considerations can be made on this crucial issue:

- Generally, it is necessary to make conservative assumptions on the loss level. Apparently, conservatism might look to be not fully compatible with a cost-benefit analysis, since it implies an excessive allocation of resources that could be better used. In reality, this is a good example of how the estimated cost must take into account not only the detriment of expected doses but also the probability and the severity of unknown events.

- Another contribution to decision making which is not easy to quantify is the knowledge based on past experience. Having already participated in the design of similar machines helps to build cheaper and more effective shielding. It is a frequent experience that the shielding of accelerators (for instance, synchrotron radiation sources) newly built for the first time in some country, fails to attain the same effectiveness that comes from a long practice.

- The use of active engineered controls such as beam loss monitors, beam intensity comparators etc., makes the issue of beam loss uncertainty less critical: but of course the cost of such devices must be included in the optimization study, as well as their possible interference with beam operation (again, something which is not easy to quantify!) 
Sometimes, the collaboration between radiation experts and accelerator engineers can result in designs which decrease cost and detriment at the same time. An example is that of proton accelerators, where radiation damage occurs mainly at points which become highly activated. "The simplest kind of decision is that in which reductions in radiation doses are accompanied by a saving of resources" $\left({ }^{11}\right)$.

Also choosing a technique for shielding calculations is a matter of optimization. Modern Monte Carlo programs are often benchmarked to predict doses within a few percent in controlled conditions. But, as in the above example of beam losses, the input conditions are not always well known, and conservative assumptions must be made. These programs are also time-consuming and expensive: in the optimization one should include not so much the cost of computers, rather cheap nowadays, but the need of specialized staff to maintain them and of Monte Carlo specialists to run the codes and to analyze the results. In alternative, the simple old-fashioned analytical programs which were used in the past are still available and can provide immediate results, at the price of a larger uncertainty of the results, hopefully on the conservative side. So, each choice has its advantages and its drawbacks, which must be quantified as monetary costs. This is an interesting example of a cost-effectiveness analysis. This type of analysis, which can be done without the need of assigning a monetary value to $\alpha$, is possible when the choice is between two options leading to a different detriment for the same cost, or between two options leading to a different cost for the same detriment, as in this case (since the target dose rates are the same).

Skyshine is an aspect of accelerator shielding design which has special implications. First, the calculated collective doses don't concern only the radiation workers, but all persons on site and especially outside the site. Dose limits and dose constraints are different for each of the three categories, but especially the economic and social factors to be considered are very different, and it is not always easy to identify how the benefit and the detriment are distributed. Must doses received by administrative staff, who gets a benefit (their salary) be weighted differently from that of the outside public, who gets no direct benefit?

But there is also a technical aspect about neutron skyshine, where again past experience plays an important role. A great deal of such past experience is collected in the book "A History of Accelerator Radiation Protection” $\left({ }^{16}\right)$. It has been a constant lesson since the '50s with the Cosmotron (Brookhaven), 
the Bevatron (Berkeley) and the Synchrophasotron (Dubna) and later Adone (Frascati), the antiproton accumulator at CERN and many other accelerators, that no accelerator of some intensity should ever be built without a roof. A cost-benefit analysis which would contradict this basic rule would need to be checked very carefully.

Another important issue in shielding design is the planned area classification and the corresponding staff dose limits and training. The choice can be in part the result of an analytical cost-benefit analysis including the cost of training and personal dosimetry, but other requirements such as ease of access may be more difficult to quantify. Also the choice between engineered and administrative controls should in principle be based on dose optimization. But the difference is difficult to quantify, being based on the comparison of the failure probabilities of the two methods, and on the corresponding dose detriment, which are not easy to express as numbers. Again, professional experience and subjective judgment will carry an important weight in the final choice.

There is an aspect of shielding design about which it may be more difficult to reach a consensus: the long term future of the facility. Shielding, especially bulk shielding, is difficult and expensive to modify, but experience shows that often the life of a research accelerator becomes extended well beyond the original plans, to a time when the technology, the use of the machine and the legislation are all completely different. A good example are the CERN PS and the SLAC Linac, which were overshielded when built, but which could not be still in use as they are if ALARA had been practiced at that time. So, if a new facility is planned at present, over how many years shall we compute the cost and the collective dose? A related issue is that of accelerator decommissioning. Modern practices (although not followed everywhere) demand that the eventual decommissioning be part of the design, but often decommissioning takes place at a time of different legislation and different technology.

\section{Operation}

At operation level, doses fall in two different categories: from prompt radiation and from residual radiation. 
Collective doses from prompt radiation are strongly influenced by the choices made in the design phase, of which we have spoken before, and concern mostly the public. In addition to skyshine, mentioned above,

radioactive releases need to be calculated and controlled. In the optimization examples provided by ICRP, the choice of ventilation systems is a recurring issue, although in general not with accelerators in mind.

On the other hand, in a well designed accelerator, worker doses are mainly from residual radiation. These, too, can be optimized in part in the design phase by the choice of shielding material: the use of marble to minimize the residual radiation in strongly activated areas is a typical choice worth of a detailed cost-benefit analysis. However, to carry such an analysis it is necessary to figure out typical intervention scenarios (how often, for how long, how many people concerned) requiring a lot of professional judgment. We stress again that the scenarios used need to be clearly documented, as well as the estimated collective dose and cost.

But another optimization will be necessary during operation. Health physicists must carefully plan all work to be performed in radioactive areas, both in routine and in exceptional cases. In everyday tasks, this is done following the health physicist professional training, where optimization is not carried out formally but implicitly. However, the formal aspect must be emphasized in written procedures to be followed in different cases.

In the case of an exceptional intervention, such as the repair of a very radioactive item, not only the collective doses but also individual dose distributions should be estimated for the sake of equity. This is the term used by ICRP to mean that no individual should receive a dose much larger than others, even though the collective dose would be about the same.

One of the choices which are easiest to optimize is the waiting time imposed before access is allowed into the accelerator enclosure. Also in this case no actual value of $\alpha$ is needed: the half-life of most radionuclides produced in air is short, so that after about one hour there will not be an appreciable further decrease of the ambient dose rate: more "cost" (actually, inconvenience) without detriment reduction. But be careful: if a much shorter delay was requested for some reason, a formal ALARA study should be made and documented. 
Dismantling and decommissioning have already been mentioned in connection with long-term ALARA planning. But the actual realization of decommissioning is a radiation protection operational task, which must to be carried out implementing appropriate RP measures to ensure that received doses are ALARA.

In a paper by $\mathrm{T}$. Labarta about dismantling of nuclear plants ${ }^{(19)}$, the author describes the ALARA management of work as follows:

"[It] is a working methodology that integrates, in a systematic and documented form, all the aspects of exposure to radiation. It develops a tree-stage method consisting of:

- Making a good plan and schedule for specific activities identifying needs, risks, protection equipment, possible incidents, etc.

- Controlling and following-up the progress of the job, implementing means to detect deviations from what was expected, and correcting them.

- Making a final analysis report to summarise lessons learned to be used as a feedback for future works: deviations from estimated activities and from estimated doses, reasons for the variations, incidents and effectiveness of corrective actions".

In all the ALARA studies here mentioned, the ALARA overhead cost itself should be included as part of the total cost.

\section{Other aspects of accelerator radiation protection}

There are other choices that the accelerator health physicist needs to make as part of his job. In general, these are not independent of other choices we have mentioned above. Decisions concerning personal dosimetry (type of dosimeter, number of persons to be monitored, etc.) are clearly connected with the classification of areas: so the cost-benefit analysis needs to take the global picture into account. On the other hand, personal dosimeters and area monitors are complementary, and their number and respective costs must be balanced against each other. Perhaps this can be done by a cost effectiveness study, assuming that the detriment (the collective dose) is the same for both monitoring systems, when the estimated dose levels are very small and the probability that a significant exposure be undetected is negligible. But it must be kept in mind that in a research accelerator the situation cannot always be so closely under control as in 
an industrial plant: for instance at some sites the presence of a large turnover of outside visitors and shortterm experiments makes it difficult to organize a dose monitoring guaranteed to be fully watertight. Such uncertainty might justify the additional cost due to more personal dosimeters or more area monitors than strictly required.

Some radiation protection issues are obvious from a professional point of view, but are not easy to quantify as required by an analytical ALARA study. For instance, choosing the proper survey instruments and having them properly maintained and calibrated is essential for a good health physics routine. But how much dose is saved by purchasing an expensive high-quality instrument rather than a cheaper one? It is clear that this is one of the many cases where professional experience weighs heavily in the cost-benefit analysis as a basis for decision which cannot be expressed as a solution of a mathematical equation. This element, however, must be documented just as well as the more quantitative ones.

We could put training in the same category.

\section{The formal part of ALARA}

As we have seen, radiation protection practices which have been established as the result of an ALARA study must be described in formally documented procedures. How they are approved and enforced (by RP, by site management or by external authorities), will depend on the national and local regulations. However, it is important that a revision scheme be made, dictating what shall trigger the preparation of a new procedure, and if necessary a new cost-benefit analysis.

A review of each existing procedure will need to be made at regular time intervals (for instance, every two years), and when one of the following "triggers" happens:

- a change in laws or regulations

- a substantial change in costs

- a substantial change in collective dose or individual dose distribution

- an abnormal occurrence of accidents or near misses. A record of such occurrences should be kept.

Funding 
This work was supported by Department of Energy contract DE-AC-03-76SF00515

\section{References}

1. Wilson R., Precautionary Principles and Risk Analysis, IEEE Technology and Society Magazine, p. 4044, Winter 2002/2003

2. ICRP 9, Recommendations of the International Commission on Radiological Protection (1966)

3. ICRP 22: Implication of Commission Recommendations that Doses be kept As Low as Readily Achievable (1973)

4. National Bureau of Standards Handbook 59: Recommendations of the National Committee on Radiation Protection (1954)

5. Nuclear Regulatory Commission, Rule Making RM-30-2 (1975). Cited by R. Wilson ( ${ }^{4}$ )

6. Clarke R. and Valentin J., A history of the International Commission on Radiological Protection, Health Phys. 88, 407-422 (2005)

7. ICRP 26, Recommendations of the International Commission on Radiological Protection, Annals of the ICRP 1, 3 (1977)

8. NCRP Report 107, Implementation of the principle As Low As Reasonably Achievable (ALARA) for medical and dental personnel (1990)

9. European Basic Safety Standards, Directive 96/29/Euratom - O.J. No L 159 of 29.6.1996

10. ICRP 37, Cost-benefit analysis in the optimization of radiation protection, Annals of the ICRP $10,2 / 3$ (1983)

11. ICRP 55, Optimisation and decision-making in radiological protection, Annals of the ICRP 20, 1 (1989) 12. ICRP 60, Recommendations of the International Commission on Radiological Protection, Annals of the ICRP 21, 1-3 (1991)

13. ICRP 103, The 2007 Recommendations of the International Commission on Radiological Protection, Annals of the ICRP 37, 2-4 (2007)

14. Sugier A., Nénot J.-C. and Lecomte J.-F., Les recommandations de la CIPR: les raisons d'un changement, Radioprotection 40, 327-344 (2005) 
15. Wiedis D., Jose D.E. and Komer K., An overview of the current state of radiation litigation, Health

Phys. 81, 253-259 (2001)

16. “A History of Accelerator Radiation Protection”, Ed. H.W. Patterson and R.H. Thomas, Nuclear Technology Publishing, Ashford, Kent (1994)

17. Labarta T., Aspects of operational radiation protection during dismantling of nuclear facilities relevant for the estimation of internal doses, Radiat. Prot. Dosim. 124, 260-265 (2007) 